\title{
Motor expertise modulates the unconscious processing of human body postures
}

\author{
Iris Güldenpenning • Dirk Koester • \\ Wilfried Kunde $\cdot$ Matthias Weigelt . \\ Thomas Schack
}

Received: 16 November 2010/ Accepted: 30 June 2011/Published online: 17 July 2011

(C) The Author(s) 2011. This article is published with open access at Springerlink.com

\begin{abstract}
Little is known about the cognitive background of unconscious visuomotor control of complex sports movements. Therefore, we investigated the extent to which novices and skilled high-jump athletes are able to identify visually presented body postures of the high jump unconsciously. We also asked whether or not the manner of processing differs (qualitatively or quantitatively) between these groups as a function of their motor expertise. A priming experiment with not consciously perceivable stimuli was designed to determine whether subliminal priming of movement phases (same vs. different movement phases) or temporal order (i.e. natural vs. reversed movement order) affects target processing. Participants had to decide which phase of the high jump (approach vs. flight
\end{abstract}

I. Güldenpenning $(\bowtie) \cdot$ D. Koester $\cdot$ T. Schack

Faculty of Psychology and Sport Science,

Neurocognition and Action - Biomechanics - Research Group,

Bielefeld University, Universitätsstraße 25,

33615 Bielefeld, Germany

e-mail: iris.gueldenpenning@uni-bielefeld.de

I. Güldenpenning · D. Koester · T. Schack

Center of Excellence-Cognitive Interaction Technology,

Bielefeld University, Universitätsstraße 21-23,

33615 Bielefeld, Germany

W. Kunde

Department of Psychology, Würzburg University,

Röntgenring 11, 97070 Würzburg, Germany

M. Weigelt

Institute of Sport Science, Saarland University,

Building B8 1, 66123 Saarbrücken, Germany

T. Schack

Research Institute for Cognition and Robotics (CorLab),

Bielefeld University, Universitätsstraße 25,

33615 Bielefeld, Germany phase) a target photograph was taken from. We found a main effect of temporal order for skilled athletes, that is, faster reaction times for prime-target pairs that reflected the natural movement order as opposed to the reversed movement order. This result indicates that temporal-order information pertaining to the domain of expertise plays a critical role in athletes' perceptual capacities. For novices, data analyses revealed an interaction between temporal order and movement phases. That is, only the reversed movement order of flight-approach pictures increased processing time. Taken together, the results suggest that the structure of cognitive movement representation modulates unconscious processing of movement pictures and points to a functional role of motor representations in visual perception.

Keywords Subliminal priming $\cdot$ Perception - Cognitive representation · High-jump photographs

\section{Introduction}

Complex actions (for example, a high-jump movement) need to be adjusted during movement execution, as not all motor commands can be determined before movement initiation (storage problem, Schmidt 1975). Importantly, movement adjustments might occur without conscious perception of relevant stimuli, which is shown in studies of clinical (Weiskrantz 1986; Perenin and Jeannerod 1975) and non-clinical (Pélisson et al. 1986; Prablanc and Martin 1992; Johnson and Haggard 2005; Chua and Enns 2005) psychology. These studies investigated, for example, adjustments made during pointing movements towards a visual target (e. g. Pélisson et al. 1986), where the target was shifted during participants' saccades and participants 
were not aware of these shifts. However, they corrected their ongoing trajectories according to the direction of the visual target shift.

To our knowledge, there is little evidence of whether or not unconscious processing of movement-related visual information is influenced by the availability of specific motor representations (e.g. for the high-jump movement). Some work has been devoted to unconsciously controlled decision-making in sports (Kibele 2006), which is particularly relevant for fast motor reactions. Imagine an elite tennis player who is attempting to return the serve of an opponent. The returning player has to decide whether he needs a forehand or a backhand return. As the flight of the ball is very fast, the player cannot change the technique once initiating a particular movement (i.e. switching between a forehand and a backhand return). Thus, decision-making processes before movement initiation play an important role for fast motor reactions.

In contrast, longer lasting and precise, to-be produced actions highly depend on adjustments made during movement execution. These adjustments require action feedback about already produced sub-goals of the movement. Think, for example, of the approach in a high jump, where the length of successive steps needs to be evaluated and adjusted in order to reach the optimal take off. Such adjustments during movement execution seem to rely on different cognitive processes than the quick decisionmaking that is done before movement initiation (e.g. decisions to play a forehand or a backhand return). Here, we are interested in the issue of whether or not unconscious visual perception of high-jump pictures is influenced by the availability of motor representations of movements that critically require adjustments during execution.

To explain visuomotor processing without awareness, Goodale et al. (1992) propose that visual information exiting the primary visual cortex (V1) follows two different 'streams'. The ventral stream travels towards the temporal lobe and is associated with object identification. The dorsal stream projects to the parietal lobe and is involved in the guidance of actions and the recognition of where objects are located in space. Assuming that information in the dorsal stream is processed independently from information in the ventral stream, a stimulus can activate a motor response via the dorsal stream, even if the ventral stream is dysfunctional. Such a motor activation may occur without conscious processing of the stimulus because conscious processing is associated with ventral stream activity. In support of this claim, unconscious motor activation has been demonstrated in patients with lesions confined to the ventral stream (Milner and Goodale 1995).

Even though unconscious processing is theoretically (Goodale and Milner 1992; Milner and Goodale 1995) and empirically (e.g. Dehaene et al. 1998; Eimer and Schlaghecken 1998) grounded, different mechanisms that may mediate this process are still widely discussed in cognitive psychology (e.g. Dehaene et al. 1998; Elsner et al. 2008; Kunde et al. 2003). A recent study suggests that domainspecific expertise is an important prerequisite of unconscious information processing (Kiesel et al. 2009). In this study, Kiesel et al. (2009) showed that chess experts can unconsciously process whether chess scenarios displayed a 'checking' or a 'non-checking' configuration, but novices cannot.

As we are interested in the role of specific motor representations in unconscious processing, we compared novices to skilled athletes of high jump regarding their perception of visual stimuli. Skilled athletes are supposed to have a particular high-jump representation at their command (for the so-called Fosbury Flop; see Method), whereas novices have no such specific motor representation. Such motor representations reflect an action-related cognitive system that is necessary for efficient movement control (Schack 2004).

A theoretical basis for the interplay between motor expertise and perceptual processes is provided by the Theory of Event Coding (TEC; Hommel et al. 2001). The core assumption of TEC is that perceived events (perceptions) and intended or to-be-generated events (actions) are represented in a common representational domain (based on the common-coding approach, see Prinz 1992; 1997). This suggestion is in accordance with the idea about a mirror neuron system in our brains (Rizzolatti 2005; Rizzolatti et al. 1996). A recent fMRI study with expert ballet dancers (Calvo-Merino et al. 2006) provides neurofunctional support for this theoretical approach. In this study, male and female dancers watched videos of dancing movements performed by both genders. As all ballet dancers train together, they have equal visual experiences for both types of dancing moves, but only motor representations for gender-specific movements. Results show that the activation of motor representations (in parietalpremotor circuits) was stronger when participants watched movements from their own movement repertoire compared to movements where they had only visual experiences. Thus, movement-related brain activity predominantly depends on specific motor knowledge and less on visual experiences, not only when performing a particular movement but also when merely observing one.

Taken together, athletes with specific motor experience have an increased perceptual sensitivity for actions in their domain of expertise (perceptual resonance; Schütz-Bosbach and Prinz 2007a) which might be the prerequisite to unconsciously processing movement information. To test this, we investigated whether novices and skilled athletes of the high-jump movement are able to identify visually 
presented body postures of the high-jump unconsciously, and whether the manner of processing differs between groups (qualitatively or quantitatively).

To this end, we conducted a subliminal response-priming experiment with photographical stimulus material taken from the high-jump movement (Fosbury Flop). In priming experiments, responding to a particular target stimulus is faster and more accurate when the target has been preceded by a prime that requires the same response (the response-congruency effect). In the present experiment, participants had to classify the target pictures as depicting either the approach or the flight phase of a highjump movement. Before the target appeared, a masked (not consciously perceivable) prime picture was presented, which could either depict a body posture from the approach or from the flight phase. It was expected that prime pictures would activate the response associated with their movement phases (see Dehaene et al. 1998) and thus influence the response to the target stimulus. This should produce a response-congruency effect, that is, faster responses when prime and target are from the same movement phase than when they are from different movement phases.

As predicting future aspects of a movement may also play a critical role in movement perception, we hypothesised that responses would be facilitated when the target depicts a future segment of the movement compared to the prime. Such an effect may occur for prime-target pairs from different movement phases. Processing should be facilitated when the prime is from the approach phase and the target is from the flight phase (natural temporal order) compared with a prime from the flight phase and a target from the approach phase (reversed temporal order). Such an effect of temporal order may also occur for prime-target pairs from the same movement phase. That is, responses should still be faster when the prime depicts a movement segment that is earlier in time than the target (within the same movement phase) compared to responses to primetarget pairs that depict a later movement segment than the target. Thus, the factors movement phases and temporal order were manipulated independently from one another. Note that the temporal distance is, on average, smaller within a movement phase than between movement phases. The factorial combination is illustrated in Fig. 1.

Because athletes are supposed to have a specific motor representation for movements within their area of skill, we predicted a response-congruency effect and an effect of temporal order for athletes. In contrast, due to a lack of a specific motor representation for the high-jump movement in novices, we predicted neither a response-congruency (or at least a smaller effect than for athletes) nor a temporalorder effect in this group.

\section{Method}

\section{Participants}

A total of 32 participants took part voluntarily and received course credit. All participants had normal or corrected-tonormal vision. They were all naive with regard to the purpose of the experiment. Before being tested, each participant provided written informed consent. The study has been performed in accordance with the ethical standards of the sixth revision (Seoul, 2008) of the 1964 Declaration of Helsinki.

Sixteen participants were assigned to the group of novices (six women, all right-handed, mean age 22.3; range 18-29) as they reported having no practical experiences in
Fig. 1 Illustration of the independently manipulated factors congruency of movement phases and temporal order. The upper line depicts prime-target pairs with a natural movement order whereas the lower line depicts prime-target pairs with a reversed movement order. This manipulation of the temporal order is true for primetarget pairs from the same movement phase (left column) and for prime-target pairs from different movement phases (right column)

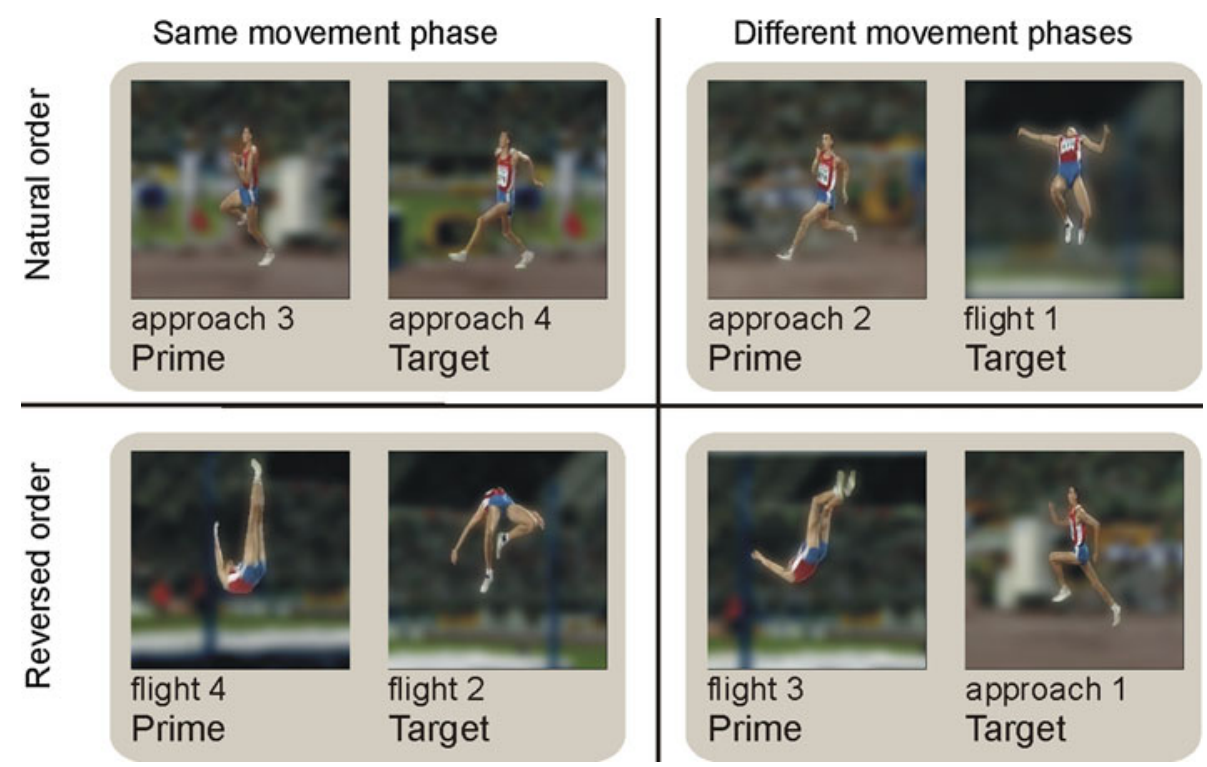


high jump or at most minimal experiences from school lessons. The group of skilled athletes consisted of sixteen participants with specific experience in high jump (10 women, one left-handed, mean age 24.1; range 19-39). Seven of the athletes were recruited from local sport clubs. Nine participants were sport students with a main focus on track and field. These students explicitly learned the technique during their semester courses and completed their courses successfully with a practical examination. Practical performance shows that the sport students have acquired a sufficient motor representation for the high-jump movement. Moreover, three of the athletes had experiences in teaching high jump. The mean training experience for the group of skilled athletes was 6.3 years (range 1-28: $\mathrm{SD}=7.1$.

\section{Apparatus and stimuli}

The stimuli were eight photographs of a high-jump movement (four approach phase images and four flight phase images) taken from a video of one final contest of the Olympic games 2008 in Peking (public television broadcasting). The whole stimulus set is depicted in Fig. 1. Two pictures of each category were used as picture primes. To rule out that priming effects are merely based on acquired mappings between targets and response keys that are also applied to subliminally presented primes, one prime picture of each category was a so-called novel prime (Abrams and Greenwald 2000; Damian 2001). Novel primes are pictures that are used as primes, but not as targets. Thus, participants never consciously perceived these pictures during the experimental session as they never saw them as a target. Therefore, participants cannot acquire a mapping between a novel prime and a response key as they never responded to the novel prime pictures.

Pre- and post-masks consisted of $25 \times 25$ randomised cut-outs $(10 \times 10$ pixels $)$ of the stimulus set and were generated automatically by visually scrambling versions of the stimuli. This was done to keep them comparable to the stimulus pictures in physical parameters, such as overall brightness and average colour (cf. Fig. 2). Stimuli and masks had a size of $9.0 \times 9.0 \mathrm{~cm}(250 \times 250$ pixels $)$. The background of the stimulus pictures was darkened and blurred to reduce distraction from irrelevant background information. All stimuli were presented centrally on a black background and subtended a visual angle of $6.5^{\circ}$ (horizontally and vertically) from the viewing distance of $80 \mathrm{~cm}$.

For stimulus presentation, an IBM-compatible computer with a 17-inch VGA-Display (vertical retraces $60 \mathrm{~Hz}$ ) and the software Presentation ${ }^{\circledR}$ was used (version 14.1; http://www.neurobs.com). The software controlled the presentation of the stimuli and measured reaction times with a precision of one millisecond.

Design and procedure

A $2 \times 2 \times 2$ mixed factorial design was used for the present study. The within-subject factors were congruency of movement phases (same phase vs. different phases) and temporal order (natural vs. reversed). Level of expertise served as a between-subject factor (skilled athletes vs. novices).

Participants sat in front of a computer screen $(80 \mathrm{~cm})$ and were instructed to classify the presented target as either an approach or as a flight image as fast and as accurately as possible. Participants had to respond by pressing one of two external buttons with their index fingers. The response button assignment was counterbalanced across subjects.

Before starting the experimental session, each participant performed 12 randomised practice trials. Each trial started with the presentation of a fixation cross (400 ms), followed by the pre-mask $(100 \mathrm{~ms})$, prime $(17 \mathrm{~ms})$, postmask (100 ms), a blank screen $(87 \mathrm{~ms})$ and the target (until the response followed; cf. Fig. 2). An inter-trial interval of 1500 ms elapsed before the next trial started. All pictures were displayed in the centre of the screen. Incorrect responses elicited a visual error feedback ("Fehler": the German word for error).

The stimulus set consisted of 24 prime-target pairs. These pairs were presented in blocks of 24 trials. These blocks were repeated ten times to avoid immediate repetitions of particular prime-target pairs. The order of appearance was randomised within each block. All trials
Fig. 2 Procedure of the experiment. The displayed example reflects a prime-target pair from different movement phases with a natural movement order
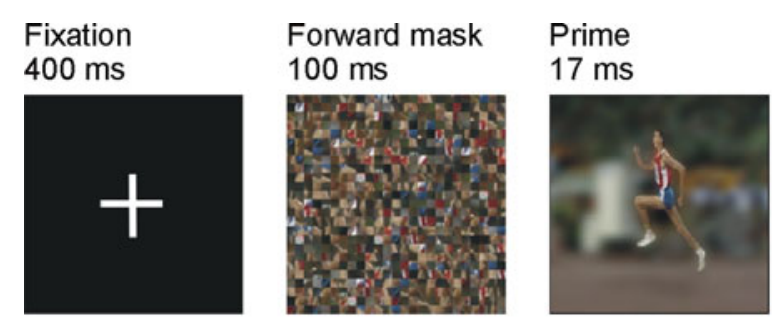

Backward mask $100 \mathrm{~ms}$

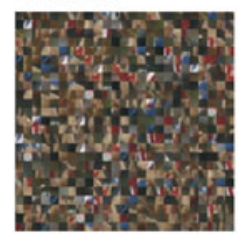

Target

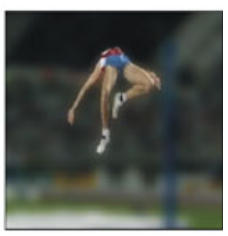

Time 


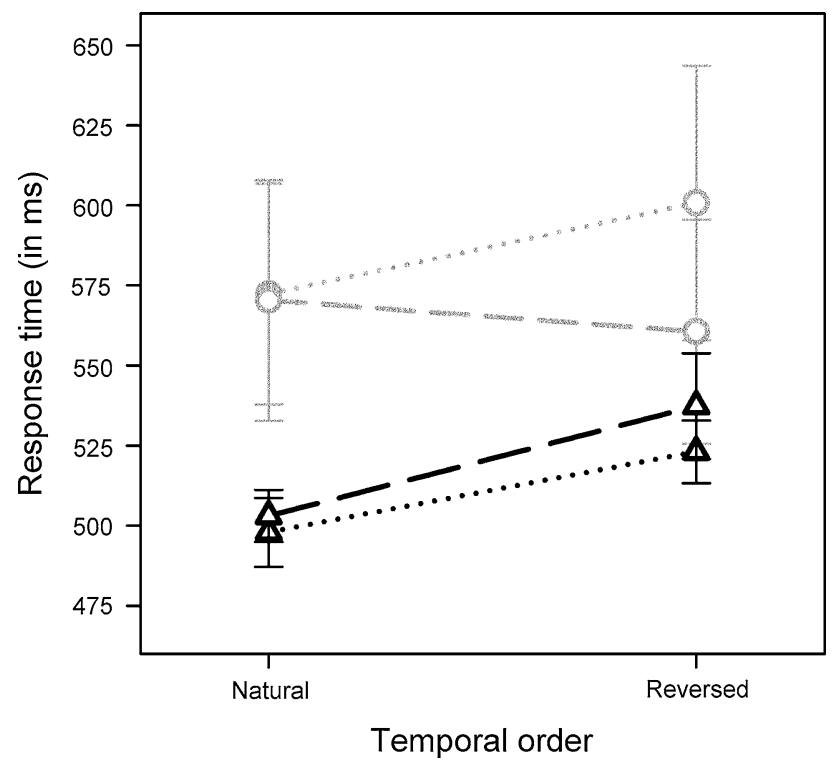

Fig. 3 Mean reaction times $(\mathrm{RT})$ in milliseconds $( \pm \mathrm{SE})$ for athletes and novices as a function of congruency of movement phases and temporal order. The black lines (triangles) illustrate RTs of skilled athletes, the grey lines (circles) the RTs of novices. The dashed lines are from congruent, the dotted lines from incongruent prime-target pairs

were presented continuously, with one short break for participants to relax.

To test whether or not the primes were consciously perceivable to the participants, a so-called detection task was performed after the experiment. Participants were fully informed about the prime stimuli and the masks and were presented with the 24 trials identical to those used during the experiment. Participants were instructed to focus their attention to the prime stimuli and were asked to classify whether the prime was of the approach or the flight phase by pressing the associated response buttons.

\section{Data analyses}

Reaction times (RTs) were screened for outliers using a total cut-off. RTs below $100 \mathrm{~ms}$ and above $2000 \mathrm{~ms}$ were excluded $(0.6 \%)$. Wrong answers $(2.9 \%)$ were not used in the RT analysis. Mean values of the RTs for each participant and condition were computed for further analyses (Fig. 3). ${ }^{1}$

\footnotetext{
${ }^{1}$ Normal distribution of the mean values for each condition within groups was tested by the Kolmogorov-Smirnov test (all $p$ 's $>0.20$ ). Furthermore, to reliably demonstrate that the measurement of central tendency (mean values vs. medians) does not influence our results, we computed the same ANOVAs with medians. These ANOVAs revealed effectively the same statistical result pattern as the ANOVAs with means.
}

A preliminary comparison of priming effects for novel primes and primes from the target set was performed to assure that priming effects were not based on acquired mappings between targets and response keys of former stimulus-response (S-R) episodes acquired during the experimental session. An ANOVA with the within-subject factor prime type (novel primes vs. primes from the target set) and the between-subject factor expertise (athletes vs. novices) revealed no main effect of prime type $(P>0.40)$ and no significant interaction of the factors prime type and expertise $(P>0.70)$. Also, the main factor expertise did not reach significance $(P>0.10)$. This result indicates that novel primes and primes from the target set did not evoke differential priming effects. Thus, we did not include prime type as an additional factor in our main analysis.

An ANOVA with the between-subject factor expertise (athletes vs. novices) and the within-subject factors temporal order (natural vs. reversed) and congruency of movement phases (same phase vs. different phases) was performed. We excluded trials with physically identical primes and targets from the congruent condition, which would artificially inflate the congruency effect due to repetition priming. The data from identical prime-target repetitions were compared to the other conditions by means of paired $t$-tests.

Error rates in the detection task were used to compute a direct measure of prime recognisability for each subject, in the form of the signal detection measure $d^{\prime}$ (Tanner and Swets 1954). Measures were obtained by treating one level of the response category factor (i.e. approach) as a signal and the other level (i.e. flight) as noise. If a participant's proportions of hits or false alarms equalled zero or one, it was corrected by using the log-linear rule (Goodman 1970; cited according to Hautus 1995).

\section{Results}

An ANOVA with the within-subject factors congruency of movement phases and temporal order and the betweensubject factor expertise was performed. The main effect of temporal order reached significance $(F(1,30)=9.18$, $\left.P<0.01, \eta_{\mathrm{p}}^{2}=0.23\right)$. The main effects congruency of movement phases and expertise did not reach significance $(P \mathrm{~s}>0.10)$. The interaction between expertise and congruency of movement phases was significant $(F(1,30)=$ 19.65, $\left.P<0.001, \eta_{\mathrm{p}}^{2}=0.40\right)$, as well as the interaction between expertise, congruency of movement phases and temporal order $\left(F(1,30)=5.39, P<0.05, \eta_{\mathrm{p}}^{2}=0.15\right)$. To follow up this interaction, we performed two ANOVAs with the factors congruency of movement phases and temporal order for novices and skilled athletes separately. 
Table 1 Mean reaction times (RT) in milliseconds and mean error rate (ER- as percentages) as a function of congruency of movement phases, temporal order and group (SEM) is given in parentheses

\begin{tabular}{|c|c|c|c|c|c|}
\hline & \multirow[t]{3}{*}{ Movement phases } & \multicolumn{4}{|c|}{ Temporal order } \\
\hline & & \multicolumn{2}{|l|}{ Natural } & \multicolumn{2}{|l|}{ Reversed } \\
\hline & & RT (SEM) & ER (SEM) & RT (SEM) & ER (SEM) \\
\hline \multirow[t]{2}{*}{ Athletes } & Same & $503(8)$ & $1.6(0.3)$ & $537(16)$ & $2.8(1.2)$ \\
\hline & Different & $498(11)$ & $2.4(0.5)$ & $523(10)$ & $2.5(0.6)$ \\
\hline \multirow[t]{2}{*}{ Novices } & Same & $570(38)$ & $2.4(0.7)$ & $561(35)$ & $5.3(2.0)$ \\
\hline & Different & $572(34)$ & $3.3(0.8)$ & 601 & $3.8(1.0)$ \\
\hline
\end{tabular}

Mean RTs (cf. Fig. 3) and error rates are illustrated in Table 1. To illustrate the variability of reaction times between the groups, frequency histograms of the reaction time data are given in Appendix 1 (skilled athletes) and Appendix 2 (novices).

For skilled athletes, the factor temporal order reached significance $\left(F(1,15)=22.29, P<0.001, \eta_{\mathrm{p}}^{2}=0.58\right)$. Responses to prime-target pairs reflecting the reversed movement order were slower $(530 \mathrm{~ms}$, SEM = 12.3) than to prime-target pairs reflecting the natural movement order $(501 \mathrm{~ms}, \mathrm{SEM}=8.7)$. The main effect of congruency of movement phases was not significant $(P<0.10)$; nor was the interaction between temporal order and congruency of movement phases $(P>0.10)$.

For novices, the factor temporal order was not significant $(P>0.10)$. The factor congruency of movement phases revealed a significant main effect $(F(1$, $\left.15)=19.96, P<0.001, \eta_{\mathrm{p}}^{2}=0.57\right)$, as did the interaction between congruency of movement phases and temporal order $\left(F(1,15)=6.77, P<0.05 ; \eta_{\mathrm{p}}^{2}=0.31\right)$. To follow up this interaction, further paired $t$-tests were performed. The results showed that only prime-target pairs from different phases with a reversed order (i.e. flight-approach pairs) led to increased RTs. These 'different phase reversed' prime-target pairs led to significantly greater RTs than 'same phase reversed' pairs $(t(15)=4.30, P<0.001)$ and 'same phase natural' pairs $(t(15)=2.81, P<0.05)$. 'Different phase reversed' pairs also led to greater RTs than 'different phase natural' pairs, but this difference only approached significance $(P<0.10)$. RTs between 'same phase natural' and 'different phase natural' pairs did not reach significance $(P>0.10)$.

The same ANOVAs on error rates revealed no significant main effects and no significant interactions.

Prime visibility

The discrimination measure for skilled athletes was $d^{\prime}=0.17$ and did not deviate significantly from zero $(t(15)=1.67, P>0.05)$. For novices, it was $d^{\prime}=-0.04$, which also did not differ significantly from zero $(t(15)=$
$-0.24, P>0.05)$. The $d^{\prime}$ values between novices and skilled athletes did not differ significantly either $(t(30)=$ $1.13, P>0.05)$.

\section{Discussion}

This study addressed the question of whether or not unconscious visual perception of high-jump pictures is influenced by the availability of motor representations. To this end, skilled athletes and novices took part in a subliminal priming study with stimulus material taken from the high-jump movement. It was predicted that categorical (i.e. movement phases) and temporal information (i.e. temporal order) would activate the motor system based on movement representations in skilled athletes but not in novices.

In accordance with our hypotheses, an effect of temporal order for skilled athletes was observed. Participants responded faster if the prime-target pairs reflected the natural order of the movement independently of whether the prime and the target were from the same movement phase or from different movement phases. The temporalorder effect is suggested to be due to activated future aspects of the perceived body posture. Thus, athletes may have anticipated the next segment of the movement when they unconsciously perceived the prime picture. Such anticipation may have facilitated encoding of a target picture, if it depicted a forthcoming action. Therefore, it is suggested that represented body postures of the high jump also contain information about future aspects that are associated with this specific movement image. Representing future states of an event is known as prospective coding (Schütz-Bosbach and Prinz 2007b).

Contrary to predictions, athletes did not show a responsecongruency effect, which means that the prime did not activate its associated motor response. The results suggest that athletes predominantly anticipated forthcoming movement segments while unconsciously processing the prime. The pre-activation of the motor response according to the prime might be subordinated to anticipation of forthcoming 
movement segments. This is remarkable, as processing of the temporal order was task-irrelevant, whereas processing of the movement phases was task-relevant. This result pattern of a temporal-order effect without a response-congruency effect for skilled athletes needs further empirical confirmation.

For novices, we found an interaction between congruency of movement phases and temporal order, indicating that only flight primes followed by approach targets slowed down the stimulus classification. In other words, novices only recognise a natural versus a reverse movement order between movement phases, but not within the approach phase or within the flight phase. This result suggests that even novices can extract at least some photographical information in a subliminal priming paradigm. It is possible that novices rely on the global orientation of a high-jump contestant (vertical vs. curved) and the athlete's position in the picture (approaching athletes tend to be in the middle, flying athletes tend to be in the upper half of the picture) which might be easier to access. It can be assumed that novices have some basic (abstract) world knowledge about the natural order of complex human movements (e.g. the approach preceding the flight phase in high jump). With this general knowledge, novices may not perceive, for example, the order of two vertically aligned contestants (approach prime + approach target picture) as a violation of the natural movement order. Similarly, a vertically oriented followed by a curved contestant, indicating an approach followed by a flight phase, adheres to the natural order of high jump. Only, the order of a curved followed by a vertically oriented contestant (flight followed by approach) may constitute a violation of the general movement order of high jump. The conflict between the perceived stimulus order and the natural movement order (contained in world knowledge) seems to slow down target processing. Thus, superficial features such as the global orientation of the contestant and their position in the picture, together with abstract world knowledge of high jump, may explain the increased reaction times for reversed prime-target pairs from different movement phases. The fact that novices did not also show a response-priming effect supports the interpretation that only the global orientation of the contestant was extracted from the primes and not the specific movement segments.

The significant increase in reaction time for flightapproach prime-target pairs for novices contrasts with the chess study of Kiesel et al. (2009), which did not find any influence of subliminally presented primes in novices. However, categorising a movement sequence and chess scenarios require different processing of relevant information. For an accurate categorisation of movement phases, participants need to recognise the orientation of the highjump athlete and their position in the picture. As knowledge about body orientation seems to be available to high-jump novices, it appears possible that novices could have accessed such information and used it in the present study. By contrast, the chess task, with a decision about a checking or a non-checking scenario, requires specific knowledge of chess rules. Since novices have little to no knowledge about chess rules by definition, it may not be possible for them to process chess scenarios (unconsciously).

We argue that the results of our study indicate that athletes as well as novices have a preferred temporal orientation of the high-jump movement sequence. Novices seem to be only aware of the fact that the movement starts with an approach phase followed by the take off and the flight. This general knowledge might be sufficient for a rough movement prediction (between movement phases). This rough movement prediction might be adequate when observing moving objects, namely to overcome the neuronal delay within the visual system (50-100 ms; cf. De Valois and De Valois 1991). This neuronal latency between sensory information take-up and perceptual information processing might be bridged by extrapolating the trajectory of a moving object at an early perceptual level (Nijhawan 1994). However, for efficient movement control during movement execution, a more precise anticipation is needed. We regard the anticipation of future states of our own body movements as anticipation of to-be-produced perceptual effects (the goal or sub-goals of the action and its consequences on the organism; ideomotor hypothesis; Greenwald 1970). As these perceptual effects allow the online control of movement execution (e.g. correcting the step length during the approach phase), movement control should be more efficient, if representation and related effect anticipation are more precise (e.g. regarding the temporal succession of different movement phases). Thus, athletes with domain-specific motor expertise may be better in movement prediction than non-athletes without domain-specific movement expertise and can make more evaluative use of the perceptual action feedback (Kunde et al. 2004; Schack 2004).

As motor responses are influenced by imperceivable primes, our study supports the suggestion that unconscious stimuli might activate a motor response via the dorsal stream without object identification in the ventral stream (Goodale et al. 1995). Thus, it can be speculated that motor performance in different sport scenarios is influenced and may be facilitated by unconscious patterns of information (e.g. body postures, perceived trajectories of moving persons or objects). It might be worthwhile to further investigate the influence of motor expertise on unconscious visuomotor control in complex sport settings, for example in team sports or competitive sports.

In conclusion, athletes as well as novices appear to have incorporated a (preferred) temporal orientation within their 
representations of human movement sequences. However, the way the movement is represented in their memory differs. Athletes' fine-tuned knowledge about the movement may contribute to more efficient movement control and thus may be fundamental in performing the movement. In contrast, novices' knowledge about the high-jump movement is based on general world knowledge. This kind of representation does not enable people to perform the high jump. Thus, different configurations of the cognitive system based on specific movement experiences qualitatively change the way in which we perceive a particular movement. Motor expertise is not a prerequisite to unconsciously processing movement-related information. Rather, motor expertise appears to modulate unconscious visual processing of body postures. Unconscious processing of temporal-order information in small steps (e.g. within a movement phase) might be an important aspect of movement control.

Conflict of interest The authors declare that they have no conflict of interest.

Open Access This article is distributed under the terms of the Creative Commons Attribution Noncommercial License which permits any noncommercial use, distribution, and reproduction in any medium, provided the original author(s) and source are credited.

\section{Appendix 1}

See Fig. 4.

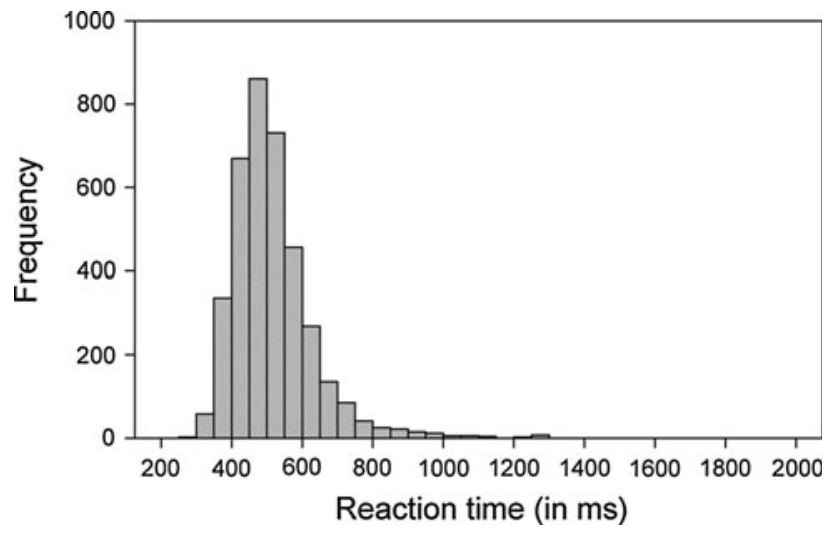

Fig. 4 Frequency histograms of the reaction times (in $\mathrm{ms}$ ) for athletes

\section{Appendix 2}

See Fig. 5.

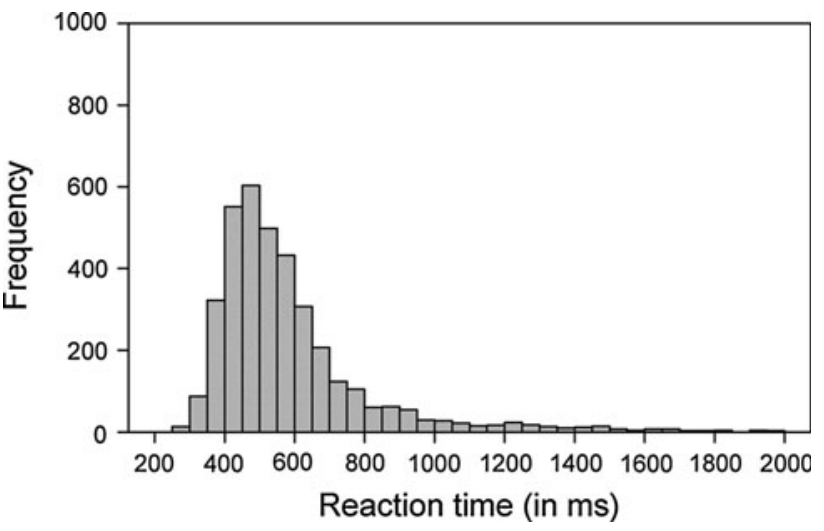

Fig. 5 Frequency histograms of the reaction times (in $\mathrm{ms}$ ) for novices

\section{References}

Abrams RL, Greenwald AG (2000) Parts outweigh the whole (word) in unconscious analysis of meaning. Psychol Sci 11:118-124

Calvo-Merino B, Grèzes J, Glaser DE, Passingham RE, Haggard P (2006) Seeing or doing? Influence of visual and motor familiarity in action observation. Curr Biol 16:1905-1910

Chua R, Enns JT (2005) What the hand can't tell the eye: illusion of space constancy during accurate pointing. Exp Brain Res 162:109-114

Damian MF (2001) Congruity effects evoked by subliminally presented primes: automaticity rather than semantic processing. J Exp Psychol Human 27:154-165

De Valois RL, De Valois KK (1991) Vernier acuity with stationary moving gabors. Vision Res 31:1619-1626

Dehaene S, Naccache L, Le Cleć HG, Koechlin E, Mueller M, Dehaene-Lambertz G, van de Moortele PF, Le Bihan D (1998) Imaging unconscious semantic priming. Nature 395:597-600

Eimer M, Schlaghecken F (1998) Effects of masked stimuli on motor activation: behavioral and electrophysiological evidence. J Exp Psychol Human 24:1737-1747

Elsner K, Kunde W, Kiesel A (2008) Limited transfer of subliminal response priming to novel stimulus orientations and identities. Conscious Cogn 17:657-671

Goodale MA, Milner AD (1992) Separate visual pathways for perception and action. Trends Neurosci 15:20-25

Goodman LA (1970) The multivariate analysis of qualitative data: interactions among multiple classifications. J Am Stat Assoc 65:226-256

Greenwald AG (1970) Sensory feedback mechanisms in performance control-with special reference to ideo-motor mechanism. Psychol Rev 77:73-99

Hautus MJ (1995) Corrections for extreme proportions and their biasing effects on estimated values of $d^{\prime}$. Behav Res Methods Instrum Comput 27:46-51

Hommel B, Müsseler J, Aschersleben G, Prinz W (2001) The theory of event coding (TEC): a framework for perception and action planning. Behav Brain Sci 24:849-937

Johnson H, Haggard P (2005) Motor awareness without perceptual awareness. Neuropsychologia 43:227-237

Kibele A (2006) Non-consciously controlled decision making for fast motor reactions in sports-a priming approach for motor responses to non-consciously perceived movement features. Psychol Sport Exerc 7:591-610 
Kiesel A, Kunde W, Pohl C, Berner MP, Hoffmann J (2009) Playing chess unconsciously. J Exp Psychol Learn 35:292-298

Kunde W, Kiesel A, Hoffmann J (2003) Conscious control over the content of unconscious cognition. Cognition 88:223-242

Kunde W, Koch I, Hoffmann J (2004) Anticipated action effects affect the selection, initiation, and execution of actions. Q J Exp Psychol A 57:87-106

Milner AD, Goodale MA (1995) The visual brain in action. Oxford University Press, Oxford

Nijhawan R (1994) Motion extrapolation in catching. Nature 370:256-257

Pélisson D, Prablanc C, Goodale MA, Jeannerod M (1986) Visual control of reaching movements without vision of the limb II: evidence of fast unconscious processes correcting the trajectory of the hand to the final position of a double-step stimulus. Exp Brain Res 62:303-311

Perenin MT, Jeannerod M (1975) Residual vision in cortically blind hemifields. Neuropsychologia 13:1-7

Prablanc C, Martin O (1992) Automatic control during hand reaching at undetected two-dimensional target displacements. J Neurophysiol 67:455-469
Prinz W (1992) Why don't we perceive our brain states? Eur J Cogn Psychol 4:1-20

Prinz W (1997) Perception and action planning. Eur J Cogn Psychol 9:129-154

Rizzolatti G (2005) The mirror neuron system and its function in humans. Anat Embryol 210:419-421

Rizzolatti G, Fadiga L, Gallese V, Fogassi L (1996) Premotor cortex and the recognition of motor actions. Cognitive Brain Res 3:131-141

Schack T (2004) The cognitive architecture of complex movement. Int J Sport Exerc Psychol 2:403-438

Schmidt RA (1975) A schema theory of discrete motor skill learning. Psychol Rev 82:225-260

Schütz-Bosbach S, Prinz W (2007a) Perceptual resonance: actioninduced modulation of perception. Trends Cogn Sci 11:349-355

Schütz-Bosbach S, Prinz W (2007b) Prospective coding in event representation. Cogn Process 8:93-102

Tanner WP, Swets JA (1954) A decision making theory of visual detection. Psychol Rev 61:401-409

Weiskrantz L (1986) Some aspects of memory functions and the temporal lobes. Acta Neurol Scand 109:69-74 